\title{
A Damage Mechanics Approach to Fatigue Assessment in Offshore Structures
}

\author{
D. L. LI \\ Department of Mechanical Engineering \\ University of Hong Kong \\ Pokfulam Road \\ Hong Kong \\ C. L. CHOw* \\ Department of Mechanical Engineering \\ University of Michigan-Dearborn \\ Dearborn, MI 48128-1491
}

\begin{abstract}
This article is intended to describe the development of a fatigue damage model capable of assessing fatigue damage in offshore structures. This is achieved by formulating a set of damage coupled constitutive and evolution equations which make the formulation of a unified approach possible under both low and high cycle fatigue damage and consistent with the structural dynamic response of the changing/deteriorating material behaviors. The structural analysis for the whole designed period, say about 30 years, can be carried out with the aid of the proposed analytical procedure, in which the fundamental characteristics of sea wave statistics responsible for the structural dynamic response can be sufficiently considered. An offshore structure subject to complex ocean environment is described by a general stochastic system which embeds a group of stochastic subsystems, each characterizing a duty cycle. An effective analytical method is established by introducing the concept of duty strain range with a clear mathematical definition and its analytical solution which covers all possible spectral parameters. The history-dependent damage is also included in the damage model so that the overload effects can be analyzed. It should be pointed out that the whole procedure can be fully computerized such that the practical or engineering significance of varying design variables can be readily highlighted.
\end{abstract}

\section{INTRODUCTION}

$\mathbf{T}$ HE DEVELOPMENT OF offshore structure and equipment for deep sea oil and gas exploitation has been motivated by an ever increasing demand for energy worldwide. It has provided an impetus to study further the mechanical behaviors

*Author to whom correspondence should be addressed. 
and failure modes of offshore structures under complex ocean environment. The thoroughgoing and painstaking approach reveals a complex interdependence among many failure modes, and identifies fatigue damage as one of main failure modes.

Fatigue damage is caused by the gradual reduction in the capacity of structural components to withstand varying service loading. However, it corresponds in physics to a rather complex mechanism characterized by the rearrangement of micro-structures of materials, which associates with an irreversible energy dissipation process. Generally, the changes in micro-structure of a material cannot be readily observed, rendering the practical difficulties in formulating the phenomena with a clear physical interpretation. Perhaps this is the main reason why for more than a century the fatigue lifetime prediction for engineering structures still relies on the empirical approach of using the Woehler's S-N curves and the Miner's law. Although this approach provides a simple engineering method in predicting the fatigue lifetime of a structure, it however fails to provide an insight into the actual failure mechanisms. Although significant advance has been made by the application of fracture mechanics, the structural dynamic response taking into account the change in mechanical behaviors of a material is yet to be portrayed. Accordingly, the damage coupled constitutive equations become necessary. These equations have been established with the theory of damage mechanics (DM) developed since the pioneering works of Kachanov [1] and Rabotnov [2].

To introduce DM into offshore engineering, the fundamental characteristics of dynamic response of offshore structures sustaining the sea wave loading will be briefly described so that admissible analytical models can be established.

\section{FUNDAMENTAL CHARACTERISTICS OF DYNAMIC RESPONSE OF OFFSHORE STRUCTURES}

In addition to the self-supporting loading, the offshore structures are continuously subjected to the complex external loading generated by surface waves over water, wind, currents and so on. The dynamic responses of an offshore structure are in general dependent on the ocean environments, the operating manners of the offshore equipment and the characteristics of the structure itself and others.

\subsection{Characteristics and Classification of Sea Wave Statistics}

The sea environment is a non-stationary stochastic process which depends on several physical parameters such as over-water wind speed, water depth, geometric conditions and so on. The process can be characterized by significant wave heights and wave periods. The structural dynamic response caused by the process has been recognized as one of the major design considerations. A realistic representation of the sea environment with the consequent loading on the structure is a highly complex problem and is still a subject of intensive experimental and theoretical research. As a fundamental treatment, the sea wave statistics are classified into short-term and long-term statistics. The former aims at general description of the sea environment during a certain interval of time in which the 
statistic properties of wave remain essentially unchanged. The latter which is decomposed into several stationary processes is oriented towards design applications for the structure with a lifetime of several years.

\subsection{Long-Term Sea Wave Statistics}

The basic data of long-term sea wave statistics can only be obtained through the in-situ monitor of ocean environment which may continue for several decades. It is a very arduous task to collect the data of sea wave statistics in the whole globe. Fortunately, the sea wave statistics for the whole globe have been inspected and collected in a data-bank [3], named as global wave statistics (GWS), and will be updated continually. These data do not refer to the special structures and cannot be utilized directly for the purpose of design. The special mathematical models and the computer software package have been developed by Chow and $\mathrm{Li}$ [4] to make these data suitable for the purpose of the structural analysis and to reproduce the sea wave processes of arbitrary periods, say about 30 years for design analysis.

\subsection{Short-Term Sea Wave Statistics and Stress Response of Structures}

The short-term sea wave statistics corresponding to the special sea states is characterized by significant wave height and dominant wave period only. Hence, it is generally independent of the sea area. The most significant characteristic of short-term statistics is that it can be rationally represented by a stationary random process. A Gaussian model for the stationary process has been shown to be a reasonable choice [5-7]. Therefore, the sea wave process can completely be described by the corresponding power spectra, such as Pierson-Moskowitz spectrum model for ocean waves

$$
S_{\eta \eta}(\omega)=\frac{H_{s}^{2} \cdot B \cdot c}{T_{D}^{c} \cdot \omega^{c+1}} \exp \left[\frac{-B}{T_{D}^{c} \cdot \omega^{c}}\right], \quad \omega>0
$$

where $T_{D}$ and $H_{s}$ denote the dominant wave period and the significant wave height, respectively; $B$ and $c$ are the spectral shape parameters; and $\omega$ is the frequency, in radians per second.

Generally, an offshore structure should be considered as a couple, multiple degrees-of-freedom system, which is governed by a set of differential equations with non-linear hydrodynamic damping and drag terms. From the standpoint of application, the linearization technique could be used so that the stress response of offshore structures corresponding to the sea wave spectrum Equation (1) can be simplified effectively. To do so, one can assume that the spectral shape of wave forces exerted on piles is unimodel and has a form similar to the wave energy spectrum. Subsequently, the wave force spectrum can be expressed as [8]

$$
S_{Q}(\omega)=\frac{Q^{2} \cdot B \cdot c}{T_{D}^{c} \cdot \omega^{c+1}} \exp \left[\frac{-B}{T_{D}^{c} \cdot \omega^{c}}\right], \quad \omega>0
$$


where $Q$ denotes the force exerted on offshore structures. Generally speaking, the stress spectrum to be represented in the form of Equation (2) will tend to be inherently conservative because it neglects reductions in energy due to spatial cancellation effects. However, for a deep water platform with fixed bottom, the first mode generally dominates the dynamic response of the structure. In this case, the stress response spectrum proposed by Wirsching and Prasthofer [8] is rational, which can be rewritten as

$$
S(f)=H_{\zeta} A \frac{\exp \left[-1050 /\left(2 \pi f T_{D}\right)^{4}\right]}{T_{D}^{4}(2 \pi f)^{5}\left\{\left[1-\left(f / f_{n}\right)^{2}\right]^{2}+\left(2 \xi f / f_{n}\right)^{2}\right\}}
$$

where $\xi$ is damping factor; $f$ denotes frequency with $f_{n}$ being the natural frequency, in Hertz; $A$ and $\varphi$ are magnification parameters.

\subsection{Local Stress-Strain Characteristics}

Fatigue failure is a complex phenomenon and is highly dependent on the local conditions of structures such as local history of stresses, micro-structure of materials, distribution of micro-flaws or cracks and so on. An accurate, at least reasonable, description of local stress history is one of the most important factors for fatigue analysis. Since offshore structures are continuously subjected to random environmental loading because of surface waves over water, wind, currents and so on, a realistic representation of the sea environment and the resulting stress on the localized area of these structures is a highly complex problem and has been the subject of intensive experimental and theoretical research.

For the tubular welded joints used for offshore platforms, the stresses and locations of hot-spots where the membrane and bending stresses have the maximum values can be determined by experiments, or evaluated by either finite element approach or empirical equations. The local effects are characterized by the stress concentration factors in saddle, $K_{s}$ and in crown, $K_{c}$, which in general depend upon the geometrical parameters such as the thickness to diameter ratio of the chord, branch diameter to chord diameter ratio, branch thickness to chord thickness ratio, the configurations of tubular joints and so on. A number of empirical or semi-empirical relations have been proposed to estimate the stress concentration factors. The comparison and analysis on these relations can be found in References [9,10]. As a function of $K_{s}$ and $K_{c}$, the hot-spot stress distribution along the weld line (hot-line stress) could be calculated by an empirical relation proposed by Dharmavasan and Dover [10]

$$
K(\beta)=K_{s} \cos ^{2} \beta+K_{c} \sin ^{2} \beta
$$

where $\beta$ denotes the angle around intersection of offshore structure, with $\beta=0^{\circ}$ at the crown position. 


\section{BASIC CONCEPTS AND FORMULATIONS OF DAMAGE MECHANICS}

The fundamental framework of DM is developed based on the irreversible thermodynamics with the internal variable theory in which the observed nonequilibrium thermodynamic state is approximated by a constrained equilibrium state corresponding to the current values of a finite set of internal variables [11-13]. The material damage characterized by a finite set of internal state variables is integrated into the thermodynamic theory. Consequently, the damage coupled constitutive and evolution equations can be derived through the thermodynamic potential and the dissipative potential which are separately postulated as the convex scalar functions of all state variables and flux variables. Due to its more complete thermodynamic framework with simpler mathematical scheme, DM has been developed to a stage ready for engineering applications [14-18]. From practical considerations, the isotropic formulation of DM is introduced although the anisotropic formulations are also possible [19-22].

\subsection{Fundamental Formulation}

According to Lemaitre's theory [14], damage represented by a scalar internal variable, $D$, is identified by the reduction of the net load carrying area due to microcavities and microcracks. The damage coupled constitutive equations are established through a strain equivalent postulation with the concept of effective stress, $\tilde{\sigma}$, defined by

$$
\tilde{\sigma}=\frac{\sigma}{1-D}
$$

With the assumption that damage is coupled through elastic response only, the thermodynamic potential $\Psi$, as dual free energy potential $\psi$, is expressed as [14]

$$
\begin{aligned}
\varrho \Psi & \left.=\left[\frac{1}{2(1-D)}\left(\frac{1+\nu}{E}<\sigma\right\rangle:\langle\sigma\rangle-\frac{\nu}{E}<\operatorname{tr}(\sigma)\right\rangle^{2}\right) \\
& \left.+\frac{1}{2(1-D h)}\left(\frac{1+\nu}{E}<-\sigma>:<-\sigma>-\frac{\nu}{E}<-\operatorname{tr}(\sigma)>^{2}\right)\right] \\
& \times\left(1-k \pi^{1 / m}\right)^{-1}-\frac{M y K y}{1+M y} p^{(1+M y) / M y}
\end{aligned}
$$

where $\pi$ denotes micro-plasticity; $p$ denotes accumulative plastic strain; $E$ and $\nu$ are elasticity coefficients varying with temperature; $\varrho$ is the density; $h$ is a closure coefficient which characterizes the closure of the micro-cracks and microcavities; $k$ and $m$ are micro-plasticity coefficients; $K y$ and $M y$ are material and 
temperature dependent coefficients. From thermodynamics, the state laws can be derived from

$$
\epsilon^{e}=\varrho \frac{\partial \Psi}{\partial \sigma} ; \quad R=-\varrho \frac{\partial \Psi}{\partial p} ; \quad r=\varrho \frac{\partial \Psi}{\partial \pi} ; \quad-Y=\varrho \frac{\partial \Psi}{\partial D}
$$

where $R, r$ and $Y$ are the associated variables of $p, \pi$ and $D$, respectively. Consequently, the elastic laws and the dual variable $Y /$ conjugate force of $D$ are derived as

$$
\begin{aligned}
\epsilon^{e} & =\frac{1}{E\left(1-k \pi^{1 / m}\right)}\left[\frac{1+\nu}{1-D}<\sigma>-\frac{\nu}{1-D}<\operatorname{tr}(\sigma)>I\right. \\
& \left.+\frac{1+\nu}{1-D h}<-\sigma>-\frac{\nu}{1-D h}<-\operatorname{tr}(\sigma)>I\right]
\end{aligned}
$$

and

$$
\begin{aligned}
-Y & =\left[\frac{(1+\nu)\langle\sigma\rangle:\langle\sigma\rangle-\nu<\operatorname{tr}(\sigma)\rangle^{2}}{2 E(1-D)^{2}}\right. \\
& \left.+h \frac{(1+\nu)<-\sigma\rangle:\langle-\sigma\rangle-\nu<-\operatorname{tr}(\sigma)\rangle^{2}}{2 E(1-D h)^{2}}\right] \frac{1}{\left(1-k \pi^{1 / m}\right)}
\end{aligned}
$$

respectively.

The complementary kinetic laws or evolution laws can be derived from a convex scalar dissipative potential $\phi$, or its dual potential $\Phi$, with the aid of the generalized normality rule;

$$
\dot{\epsilon}^{P}=\frac{\partial \Phi}{\partial \sigma} ; \quad R=-\frac{\partial \Phi}{\partial \dot{p}} ; \quad \dot{D}=-\frac{\partial \Phi}{\partial Y} ; \quad \sigma=\frac{\partial \Phi}{\partial \dot{e}^{P}} ; \quad r=-\frac{\partial \Phi}{\partial \dot{\pi}}
$$

A simple dissipative potential, only with its two coefficients characteristic of the material, $\mathrm{S}_{0}$ and $\alpha_{0}$, suggested by Lemaitre [14] has the form

$$
\Phi\left(Y, \dot{p}, \dot{\pi} ; T, \epsilon^{e}, D\right)=\frac{Y^{2}(\dot{p}+\dot{\pi})}{2 \mathrm{~S}_{0}(1-D)^{\alpha 0}}
$$

This yields a general damage kinetic constitutive equation as

$$
\dot{D}=-\frac{\partial \Phi}{\partial Y}=\frac{-Y(\dot{p}+\dot{\pi})}{S_{0}(1-D)^{\alpha 0}}
$$


This equation may reflect some properties of damage, as pointed by Lemaitre [14], such as

- Damage rate is always positive because $-Y, \dot{p}$ and $\dot{\pi}$ are positive.

- The variable $-Y$ containing the triaxiality ratio $\left(\sigma_{\mathrm{H}} / \sigma_{\mathrm{eq}}\right)$ models the effect of triaxiality on damage.

- If $-Y$ is calculated from the quasi-unilateral expression, it can model the difference in the damage behavior in tension and compression and also mean stress effects on fatigue damage.

- The non-linearity of damage with regard to stress is given by its dependence on $\dot{p}$ and $\dot{\pi}$ which are non-linear functions of stress.

- The non-linearity of damage with regard to number of cycles or time is given by the term $(1-D)^{\alpha 0}$ which corresponds to a non-linear differential equation in damage.

\subsection{Formulation for Fatigue Damage}

According to Lemaitre, the formulations for fatigue damage are divided into low cycle fatigue and high cycle fatigue. For low cycle fatigue, the plastic strain involved is high enough to be measured macroscopically. Accordingly, the formulation of damage evolution is based on the evolution of plastic strain or accumulative plasticity. From Equation (12) and the Ramber-Osgood strain hardening law, the damage evolution law is derived as

$$
\dot{D}=\left[R_{v}\left(p-p_{i}\right)^{\gamma} \cdot \dot{p}\right] /\left[S_{1}(1-D)^{\alpha 1}\right]
$$

where $S_{1}$ and $\alpha_{1}$ are two material coefficients; $\gamma=2 / M_{c}$ with $M_{c}$ being the coefficient of Ramber-Osgood law; $p$ denotes the accumulating plasticity with $p_{i}$ being the value of $p$ at the beginning of cycle $i$. $R_{v}$ is the triaxiality function which in pure bilateral condition can be expressed as

$$
R_{v}=2(1+\nu) / 3+3(1-2 \nu)\left(\sigma_{\mathrm{H}} / \sigma_{\mathrm{eq}}\right)^{2}
$$

in which $\sigma_{\mathrm{H}}$ is the hydrostatic stress and $\sigma_{\mathrm{cq}}$ denotes the Von Mises equivalent stress.

The cyclic constitutive equation is obtained by integrating Equation (13). A simple formulation can be derived as

$$
\delta D / \delta N=\left(R_{\nu} \Delta p^{\gamma+1}\right) /\left[S_{1}(\gamma+1)(1-D)^{\alpha 1}\right]
$$

under the assumptions of $R_{v}$ and $(1-D)^{\alpha 1}$ being constants over one cycle.

When the plastic strain in high cycle fatigue is assumed to be small rendering difficulty in the macro-measurement and modeling, the formulation for damage evolution may be changed to the stress space. The general formulation (13) in conjunction with a stress relative micro-plastic model leads to [14]

$$
\dot{D}=\left[\sigma_{\mathrm{eq}}^{2}\left|\sigma_{\mathrm{eq}}-\bar{\sigma}_{\mathrm{eq}}\right|^{\lambda} / S_{2}\right] /\left[R_{\nu}\left|\dot{\sigma}_{\mathrm{eq}}\right| /(1-D)^{\alpha 2}\right]
$$


where $S_{2}, \alpha_{2}$ and $\lambda$ are material coefficients. The formulation could also be integrated to obtain the cyclic formulation [14].

\section{GENERAL ANALYTICAL PROCEDURE}

Since coupled constitutive laws for stress, strain and damage have been established by DM, the damage assessment in conjunction with the stress/strain analysis of a structure can be carried out by virtue of the numerical methods, such as those proposed by Chow and Wang [23] and Simo and Ju [18]. Theoretically, a continuous analysis for the non-linear damage response in conjunction with the effect of stress triaxiality, overloading induced by random sea wave process and so on, can be achieved because complete stress response for the structure over the entire period designed for service, say about 30 years, can be simulated by a fully computerized numerical method [4]. In practice, this analysis is prohibitive in considering the limitations of numerical calculation with respect to computer speed and practical difficuities in the experimental identification for millions of different sea waves. Accordingly, an admissible analytical procedure with suitable simplifications is still necessary.

A general analytical procedure proposed here is based on the fundamental characteristics of sea wave statistics. Classification of the sea wave process requires that the structural dynamic response, coupled with changing material behaviors, be divided as short-term and long-term responses. Consequently, the general procedure can be carried out analytically by two steps as follows.

The first step is to analyze the fatigue damage induced by the short-term stress response represented by the stress power spectrum shown in Equation (3) including the effect of local stress concentration at hot-spots. The damage analysis includes all possible responses for different sea states characterized by different wave heights and wave periods. The analysis could be carried out in the manner of cycle by cycle within the characteristic periods so that the detail results can be obtained. It is possible since the analytical method for fatigue damage, the experimental identification for the practical response as well as the computer simulation for the special stress response are all available. However, this analysis will be very tedious, so it should be avoided except to meet the practical requirement. A simple model to be developed in the following two sections can provide an effective analytical method for the step.

The second step refers to the general dynamic response corresponding to the long-term sea wave statistics. The damage response to be analyzed in the step determined by a stochastic Markov process with transition probability being identical with that of long-term sea wave statistics, and hence can be analyzed and simulated using the method and software developed by Chow and Li [4]. The damage evolution is also dependent on the sea state determined by the Markov process, which is treated as a stochastic subsystem embedded in a general stochastic system represented by long-term statistics. The interactions among different subsystems could be portrayed if experimental identification for these interactions is possible. A simple model described in Section 7 enables both experimental identification and theoretical analysis to be realized. 


\section{A UNIFIED FORMULATION FOR BOTH LOW AND HIGH CYCLE FATIGUES}

As a common practice, fatigue damage is, in general, divided into low cycle and high cycle ones represented by an obscure definition; the number of cycles to failure, $N_{R}$, less than $10^{4}$ as low cycle one; $N_{R}$, more than $10^{5}$ as high cycle one. There is no clear definition for $10^{4}<N_{R}<10^{5}$. By following the division, different formulations for low and high cycle fatigues have been established. For example, Lemaitre's damage evolution laws for low and high cycle fatigues are formulated in strain and stress spaces respectively. In practical applications, particularly for offshore structures subjected to random sea wave loading, difficulties arise in identifying and distinguishing these two kinds of fatigue, and hence the choice of a formulation. In fact, the division of fatigue between low and high cycle is not clear cut and there exists no dependable physical judgment to support this division. Perhaps the only difference is that low cycle fatigue is normally associated with a significant amount of observable plastic deformation while high cycle fatigue is not, rendering the difficulty in both parametric and macro-mechanics identification. To overcome this problem, a new method based on the concepts of duty cycle and duty strain range is proposed. This leads to a modified formulation of Equation (15) expressed as

$$
\delta D / \delta C_{u}=\left(R_{v} \Delta \epsilon_{u}^{\mathrm{c}}\right) /\left[S_{3}(\gamma+1)(1-D)^{\alpha 1}\right]
$$

where $\mathbf{c}=\gamma+1$. In contrast to Equation (15), the number of cycle, $N$, has been replaced by the number of duty cycle $C_{u}$ which is to be defined and discussed later; and the accumulating plasticity $\Delta p$ has been replaced by duty strain range $\Delta \epsilon_{u}$ with the mathematical definition and solution to be presented in the following subsection. This modification renders a small change in material coefficient $S_{1}$, written as $S_{3}$. The physical and thermodynamic fundamentals for using the unified formulation to describe the whole fatigue process are established through a physical-macro-mechanics-probabilistic consistent approach [24-31].

The duty cycle $C_{u}$ introduced in Equation (17) is defined as a characteristic quantity of one time transition of sea wave states determined by the long-term wave statistics. This definition includes three implications as follows:

1. Duty cycle itself corresponds to a damage accumulating process induced by a stationary random process, but not a single loading cycle.

2. In one duty cycle, damage is treated as a process of non-ageing effect, that is, the damage process is only dependent on the current damage and strain states. Therefore, the damage process in one duty cycle can be viewed as a stationary Markov process.

3. Duty cycle denotes only a repetitive quantity, but not a characteristic of damage. Only are two states referring to the start and end of a duty cycle important for the whole damage process.

It is obvious that the introduction of duty cycle leads to two significant advantages. One is that the simple mathematical formulation for strain response which 
controls the damage response is possible since the sea wave response in one duty cycle could be treated as a stationary stochastic process represented by the power spectrum [see Equation (3)]. Another is that the damage identification for both low and high cycle fatigue (in fact, this division has been nullified) is possible simply because the damage in one duty cycle corresponds to the results of accumulation.

It should be noted that the whole damage process is characterized by a Markov process in relation to the long-term sea wave statistics, and the accumulation of damage is determined by the duty strain ranges in relation to the duty cycle in which another Markov process is included.

\section{DUTY STRAIN RANGE AND ITS SOLUTIONS}

Associated with the concept of duty cycle, another characteristic quantity in relation with the dynamic response to be introduced is the duty strain range. The word "duty" associated with quantity, cycle and strain range refers to responsibility and equivalence which are often used in the theory of probability and system engineering.

\subsection{Definition of Duty Strain Range}

There exist many methods to define a duty strain range which could be used to characterize the strain response induced by the short-term sea wave statistics. The simplest method is to relate it to the distribution of strain peaks and assume that the stochastic process in one duty cycle is one of non-ageing effects. It implies that the interaction of sea waves in one duty cycle induces only a shift of the mean value of damage state. Consequently, the duty strain range can be defined as the mean value of all strain ranges considered. Furthermore, the non-linear relation between the strain range and damage can also be established through the definition of the duty strain range. For the damage evolution laws describing the nonlinear relation of strain and damage with the power function, Equation (17), by means of the constant $\mathbf{c}$, the duty strain range $\epsilon_{u}$ can be expressed similar to the equivalent stress range used in Reference [35]:

$$
\Delta \epsilon_{u}=\left[\frac{\sum\left(\Delta \epsilon_{i}\right)^{\mathrm{c}} \cdot\left(n_{t}\right)}{\Sigma n_{i}}\right]^{1 / \epsilon}=\left[\int_{0}^{\infty}(2 \epsilon)^{\mathrm{t}} \cdot \mathfrak{p}(\epsilon) \cdot d(\epsilon)\right]^{1 / \mathrm{c}}
$$

where $\epsilon$ denotes the strain level of peak with $\Delta s_{i}$ identifying the strain range of $i$ th level of amplitude, and $n_{i}$ denotes the number of cycles corresponding to $\Delta \epsilon_{i}$. $\mathbf{p}(\epsilon)$ is the probability density of $\epsilon$, which could be expressed by means of the spectral parameters, effective band width $\mathfrak{e}$ and irregularity factor $\mathfrak{a}$.

\subsection{Probabilistic Description of Duty Strain Range}

According to the probabilistic theory, the peak distribution of a continuous random process, $x(t)$, can be obtained by means of the joint distribution of $x(t)$, 
$\dot{x}(t)$, and $x^{\prime \prime}(t)$ if $x(t)$ is differentiable at least twice. Assuming the expected number of peaks in $x(t)$ above the level $s$ is bounded, the mean value of $s$ at time $t$ can be expressed as

$$
\mathrm{E}[s(t)]=-\int_{1}^{\infty} d x \int_{-\infty}^{0} \ddot{x} \cdot \mathfrak{p}_{1 \ldots i n i n}(x, 0, \ddot{x}, t) d \ddot{x}
$$

where $\boldsymbol{p}_{|x||\dot{x}|\{\dot{x}}(\cdot)$ denotes the joint distribution of $x(t), \dot{x}(t)$, and $\ddot{x}(t)$. The expected total number of peaks per unit time, regardless of their magnitudes, is obtained from Equation (19) by letting $s \rightarrow-\infty$, that is

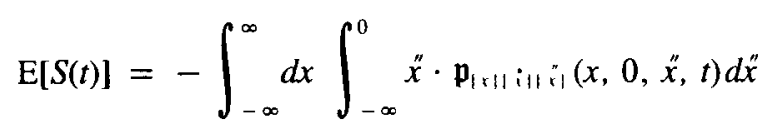

If the ratio of the expected number of peaks per unit time $(E[S(t)]-E[s(t)]) /$ $E[S(t)]$ is postulated to be equal to the distribution of the peaks at time $t$, i.e.

$$
\mathrm{p}(s, t)=(E[S(t)]-E[s(t)]) / E[S(t)]=1-\frac{E[s(t)]}{E[S(t)]}
$$

then the probability density of the stress peaks can be obtained by

$$
\begin{aligned}
\mathfrak{p}(s, t) & =\frac{\partial \mathfrak{p}(s, t)}{\partial s}=-\frac{1}{E[S(t)]} \cdot \frac{\partial}{\partial s} E[s(t)] \\
& =\frac{-1}{E[S(t)]} \int_{-\infty}^{0} \ddot{x} \cdot \mathfrak{p}_{1 \ldots i \ldots i}\left(x, 0, x^{\prime \prime}, t\right) d x^{\prime \prime}
\end{aligned}
$$

If $x(t)$ is stationary and Gaussian and has a zero expectancy, then the probability density of the peaks can be derived in terms of the matrix of covariances of $x(t), \dot{x}(t)$, and $\ddot{x}(t)$ which is not difficult to solve [32]. However, a simpler solution, suggested by Huston and Skopinski [33], reads

$$
\begin{aligned}
\mathfrak{p}(s) & =\frac{1}{\sqrt{(2 \pi) \cdot \boldsymbol{d}}} \sqrt{ }\left(1-\mathfrak{a}^{2}\right) \cdot \exp \left\{-s^{2}\left[s \grave{\mathbf{d}}^{2}\left(1-\mathfrak{a}^{\alpha}\right)\right]^{-1}\right\} \\
& +\frac{s}{2 \boldsymbol{d}^{2}} \mathfrak{a} \cdot\left[1+\operatorname{erf}\left[\frac{s}{\mathfrak{d} \cdot \sqrt{\left(2 \cdot \mathfrak{a}^{-2}-2\right)}}\right]\right] \cdot \exp \left(\frac{-s^{2}}{2 \boldsymbol{d}^{2}}\right)
\end{aligned}
$$


By putting

$$
s=x 內 e \sqrt{ } 2 / a
$$

Equation (23) can be simplified to [34]

$$
\begin{aligned}
\mathfrak{p}(x) & =\frac{\mathfrak{e}}{\sqrt{(2 \pi) \cdot \boldsymbol{d}}} \exp \left[-\left(\frac{x}{\mathrm{a}}\right)^{2}\right] \\
& +\frac{x \mathbf{e}}{\sqrt{(2) \cdot \mathfrak{d}}}[1+\operatorname{erf}(x)] \cdot \exp \left[-\left(\frac{x \mathfrak{R}}{\mathrm{a}}\right)^{2}\right]
\end{aligned}
$$

in which the error function (or probability integral) is defined as

$$
\operatorname{erf}(x)=\frac{2}{\sqrt{(\pi)}} \int_{0}^{\infty} \exp \left(-z^{2}\right) d z
$$

Equation (25) is a general expression for the probability density of the peaks of a stationary and Gaussian random process. In two extreme cases corresponding to 0 and 1 of a, Equation (25) will reduce to the Rayleigh and Gaussian distributions, respectively, i.e.

$$
\mathfrak{p}(s)=\frac{1}{\sqrt{(2 \pi \boldsymbol{D})}} \exp \left(\frac{-s^{2}}{2 \boldsymbol{d}^{2}}\right)
$$

for $-\infty<s<\infty$ and $\boldsymbol{a}=0$, and

$$
\mathfrak{p}(s)=\frac{s}{\boldsymbol{d}^{2}} \exp \left(\frac{-s^{2}}{2 \boldsymbol{J}^{2}}\right)
$$

for $0<s<\infty$ and $\mathfrak{a}=1$. Except in these two extreme cases, the distribution of peaks of a stationary Gaussian random process is neither of Rayleigh nor of Gaussian type.

Using Equation (25) and with $s=\epsilon$, the duty strain range defined by Equation (18) can be rewritten as

$$
\Delta \epsilon_{u}=2 \sqrt{2} d \cdot\left[\frac{\mathbf{e}^{\mathrm{c}+2}}{2 \sqrt{(\pi)}} \Gamma\left(\frac{\mathfrak{c}+1}{2}\right)+\frac{\mathfrak{a}}{2} \Gamma\left(\frac{\mathfrak{c}+2}{2}\right)+\mathbf{a} \cdot \zeta\right]^{1 / c}
$$

where the function $\zeta$ has the form

$$
\zeta=\int_{0}^{\infty} \operatorname{erf}(x) \cdot\left[\frac{\mathrm{e} x}{\mathrm{a}}\right]^{m+1} \exp \left[-\left(\frac{\mathbf{e x}}{\mathbf{a}}\right)^{2}\right] d\left(\frac{\mathbf{e x}}{\mathbf{a}}\right)
$$


and Gamma function is defined as

$$
\Gamma(z)=2 \int_{0}^{\infty} y^{2 z-1} \exp \left(-y^{2}\right) d y
$$

for $z>0$.

\subsection{Series Resolution of Duty Strain Range}

In general, the exact resolution for the duty strain range expressed by Equation (29) is quite difficult to be obtained since this equation contains a transcendental function. Therefore, a solution by virtue of asymptotic and series expansions will be resorted to in the present study.

By means of Maclaurin expansion, the error function, erf $(x)$, can be expressed as

$$
\operatorname{erf}(x)=\frac{2}{\sqrt{(\pi)}} \sum_{k=0}^{\infty} \frac{(-1)^{k} \cdot x^{2 k+1}}{k ! \cdot(2 k+1)}
$$

This expansion leads to an alternative form of Equation (30), that is

$$
\begin{aligned}
\zeta & =\frac{2}{\sqrt{(\pi)}} \sum_{k=0}^{\infty} \frac{(-1)^{k}}{k ! \cdot(2 k+1)} \int_{0}^{\infty} x^{2 k+1}\left[\frac{\mathfrak{e} \cdot x}{\mathfrak{a}}\right]^{\mathfrak{c + 1}} \cdot \exp \left[-\left(\frac{\mathfrak{e} \cdot x}{\mathfrak{a}}\right)^{2}\right] d\left(\frac{\mathfrak{e} \cdot x}{\mathfrak{a}}\right) \\
& =\frac{1}{\sqrt{(\pi)}} \sum_{k=0}^{\infty} \frac{(-1)^{k}}{k ! \cdot(2 k+1)} \cdot\left(\frac{\mathfrak{a}}{\mathfrak{e}}\right)^{2 k+1} \cdot \Gamma\left(\frac{\mathfrak{c}+2 k+3}{2}\right)
\end{aligned}
$$

Consequently, the series solution for the duty strain range is obtained as

$$
\begin{aligned}
\Delta \epsilon_{u} & =2 \sqrt{(2) \mathfrak{d}}\left[\frac{\mathfrak{e}^{\mathfrak{c}+2}}{2 \sqrt{(\pi)}} \Gamma\left(\frac{\mathfrak{c}+1}{2}\right)+\frac{\mathfrak{a}}{2} \Gamma\left(\frac{\mathfrak{c}+2}{2}\right)\right. \\
& \left.+\frac{\mathfrak{a}}{\sqrt{(\pi)}} \sum_{k=0}^{\infty} \frac{(-1)^{k}(\mathfrak{a} / \mathfrak{e})^{2 k+1}}{k ! \cdot(2 k+1)} \Gamma\left(\frac{\mathfrak{c}+2 k+3}{2}\right)\right]^{1 / \mathfrak{t}}
\end{aligned}
$$

An alternative solution can also be derived by using a series expression. By virture of series expansion, $\operatorname{erf}(x)$ can be expressed as

$$
\operatorname{erf}(x)=\frac{2}{\sqrt{(\pi)}} \exp \left(-x^{2}\right) \cdot \sum_{k=0}^{\infty} \frac{2^{k} \cdot x^{2 k+1}}{(2 k+1) ! !}
$$


for $|x|<\infty$. Substituting Equation (35) into Equation (30) yields

$$
\zeta=\frac{2}{\sqrt{(\pi)}}(\mathfrak{e} / \mathfrak{a})^{\mathrm{c}+2} \sum_{k=0}^{\infty} \frac{2^{k}}{(2 k+1) ! !} \int_{0}^{\infty} x^{2 k+++2} \exp \left[-x^{2}-(x \mathfrak{e} / \mathfrak{a})^{2}\right] d x
$$

Since

$$
\left[1+(\mathfrak{e} / \mathfrak{a})^{2}\right]^{1 / 2}=1 / \mathfrak{a}
$$

then Equation (36) becomes

$$
\begin{aligned}
\zeta & =\frac{2}{\sqrt{(\pi)}}(\mathbf{e} / \mathfrak{a})^{\kappa+2} \sum_{k=0}^{\infty} \frac{2^{k} \cdot \mathfrak{a}^{2 k+\mathfrak{c}+3}}{(2 k+1) ! !} \int_{0}^{\infty}(x / \mathfrak{a})^{2 k+c+2} \exp \left[-(x / \mathfrak{a})^{2}\right] d(x / \mathfrak{a}) \\
& =\frac{\mathbf{e}^{r+2}}{\sqrt{(\pi)}} \cdot \sum_{k=0}^{\infty} \frac{2^{k} \cdot \mathfrak{a}^{2 k+1}}{(2 k+1) ! !} \cdot \Gamma\left(\frac{2 k+\mathfrak{c}+3}{2}\right)
\end{aligned}
$$

Consequently, another solution of the duty strain range can be expressed in the series form as

$$
\begin{aligned}
\Delta \epsilon_{u} & =2 \sqrt{(2) \mathfrak{d}}\left[\frac{\mathfrak{e}^{\mathfrak{c}+2}}{2 \sqrt{(\pi)}} \Gamma\left(\frac{\mathfrak{c}+1}{2}\right)+\frac{\mathfrak{a}}{2} \Gamma\left(\frac{\mathfrak{c}+2}{2}\right)\right. \\
& \left.+\frac{\mathfrak{e}^{\mathrm{c}+2}}{\sqrt{(\pi)}} \cdot \sum_{k=0}^{\infty} \frac{2^{k} \mathfrak{d}^{2 k+2}}{(2 k+1) ! !} \Gamma\left(\frac{2 k+\mathfrak{c}+3}{2}\right)\right]^{1 / \mathfrak{c}}
\end{aligned}
$$

The solutions of duty strain range, Equations (34) or (39), are based on the series expansion of error function in the integral Equation (30). By means of D'Alembert method of series convergency, it can be readily proved that Equation (39) is convergent with any set of the statistical parameters (e,a) and Equation (34) is only convergent with finite values of $(\boldsymbol{e}, \mathfrak{d})$. The maximum absolute values of truncated error of (32), according to Leibniz convergence principle, can be calculated from

$$
E_{m} \leq \frac{1}{\sqrt{(\pi)} \cdot(k+1) ! \cdot(2 k+3)} \cdot x^{2 k+3}
$$

by taking the first $k$ term as its solution. It is obvious that the solution is not suitable for large $x$ because the number of terms needed to obtain a certain number of significant digits increases rapidly as $x$ increases. For a general solution capable of covering all possible statistical parameters, a complementary solution is necessary. 


\subsection{Asymptotic Solution of Duty Strain Range}

In contrast with the series solutions, the solution based on the asymptotic expansion is specially suitable for larger $x$. It means that it needs only the fewer terms of an asymptotic expansion to obtain a required accuracy. From Equation (24), $x$ will tend to infinity when $\mathfrak{e}$ approaches to zero, corresponding to the circumstances near the narrow band spectra. In these cases, an asymptotic solution is necessary.

The asymptotic expansion of Equation (26) can be expressed in the form as

$$
\operatorname{erf}(x)=1-\exp \left(-x^{2}\right) \frac{1}{\sqrt{(\pi) x}}\left[1+\sum_{k=1}^{\infty} \frac{(-1)^{k}(2 k-1) ! !}{\left(2 x^{2}\right)^{k}}\right]
$$

Consequently, the asymptotic solution of duty strain range is

$$
\begin{aligned}
\Delta \epsilon_{u} & =\left(2 \sqrt { ( 2 ) \mathbb { d } ) } \left[\frac{\mathfrak{e}^{\mathfrak{c}+2}}{2 \sqrt{ }(\pi)} \Gamma\left(\frac{\mathfrak{c}+1}{2}\right)+\mathfrak{a} \Gamma\left(\frac{\mathfrak{c}+2}{2}\right)-\frac{\mathfrak{e}^{\mathfrak{c}+2}}{2 \sqrt{ }(\pi)} \Gamma\left(\frac{\mathfrak{c}+1}{2}\right)\right.\right. \\
& \left.-\frac{\mathfrak{e}^{\mathfrak{c}+2}}{2 \sqrt{(\pi)}} \cdot \sum_{k=}^{\infty} \frac{(-1)^{k}(2 k-1) ! !}{\left(2 \mathfrak{a}^{2}\right)^{k}} \Gamma\left(\frac{\mathfrak{c}-2 k+1}{2}\right)\right]^{1 / \mathfrak{c}}
\end{aligned}
$$

with the maximum truncated error to be determined by

$$
E_{m} \leq \frac{\exp \left(-x^{2}\right) \cdot(2 k-1) ! !}{2^{k} \cdot \sqrt{(\pi)} \cdot x^{2 k+1}}
$$

for $x>0(k=1,2, \ldots)$. It is also obvious that $E_{m}$ rapidly decreases with the increase in $x$. Accordingly, this solution is suitable for the conditions of small $\mathfrak{e}$.

\subsection{Combination of Series and Asymptotic Solutions}

Theoretically, a solution of the duty strain range with any degree of accuracy for the statistical parameters $(\mathfrak{e}, \mathfrak{a})$ is attainable by the combined use of the series and asymptotic solutions if the number of terms $k$ tends to be infinite. Practically, it is not necessary to taking $k \rightarrow \infty$ since a certain degree of accuracy is acceptable from the standpoint of an engineering solution. A solution with a finite number of the $k$-terms is therefore derived as

$$
\begin{aligned}
\Delta \boldsymbol{\epsilon}_{u} & =2 \sqrt{(2) \mathfrak{d}}\left[\frac{\mathfrak{e}^{\mathfrak{c}+2}}{2 \sqrt{(\pi)}} \Gamma\left(\frac{\mathfrak{c}+1}{2}\right)+\frac{\mathfrak{a}}{2} \Gamma\left(\frac{\mathfrak{c}+2}{2}\right)\right. \\
& \left.+\frac{\mathfrak{e}^{\mathfrak{c}+2}}{\sqrt{(\pi)}} \cdot \sum_{k=0}^{k !} \frac{2^{k} \mathfrak{a}^{2 k+2}}{(2 k+1) ! !} \Gamma\left(\frac{2 k+\mathfrak{c}+3}{2}\right)\right]^{1 / \mathfrak{c}}
\end{aligned}
$$


for $\mathfrak{e} \geq \mathfrak{e l}$ and

$$
\begin{aligned}
\Delta \epsilon_{u} & =2 \sqrt{(2) \mathfrak{d}}\left[\frac{\mathfrak{e}^{\mathfrak{c}+2}}{2 \sqrt{(\pi)}} \Gamma\left(\frac{\mathfrak{c}+1}{2}\right)+\mathfrak{a} \Gamma\left(\frac{\mathfrak{c}+2}{2}\right)\right. \\
& \left.-\frac{\mathfrak{e}^{\mathfrak{c}+2}}{2 \sqrt{(\pi)}} \Gamma\left(\frac{\mathfrak{c}+1}{2}\right)+\frac{\mathfrak{e}^{\mathfrak{c}+2}}{4 \sqrt{(\pi)} \cdot \mathfrak{a} \cdot \mathfrak{a}} \Gamma\left(\frac{\mathfrak{c}-1}{2}\right)\right]^{1 / \mathfrak{c}}
\end{aligned}
$$

for $\mathfrak{e}<\mathfrak{e} 1$, where $\mathfrak{e} 1$ and $k 1$ can be determined automatically with the aid of a simple computer program by following the methodology described in Figure 1 when the expected accuracy for duty stress range is specified as input. The algorithm and formulation are the same as those described in Reference [35] for the fatigue damage assessment using Paris crack growth law. Equations (44a) and (44b) are a unified formulation for the duty strain range as a function of the strain or stress power spectrum associated with all possible spectral parameters. The accuracy of calculation with only a few terms is sufficient to satisfy the practical requirement. The comparison with several empirical and semiempirical models proposed for a similar objective has been performed by Chow and Li [35]. Excellent results as well as their practical significances were highlighted.

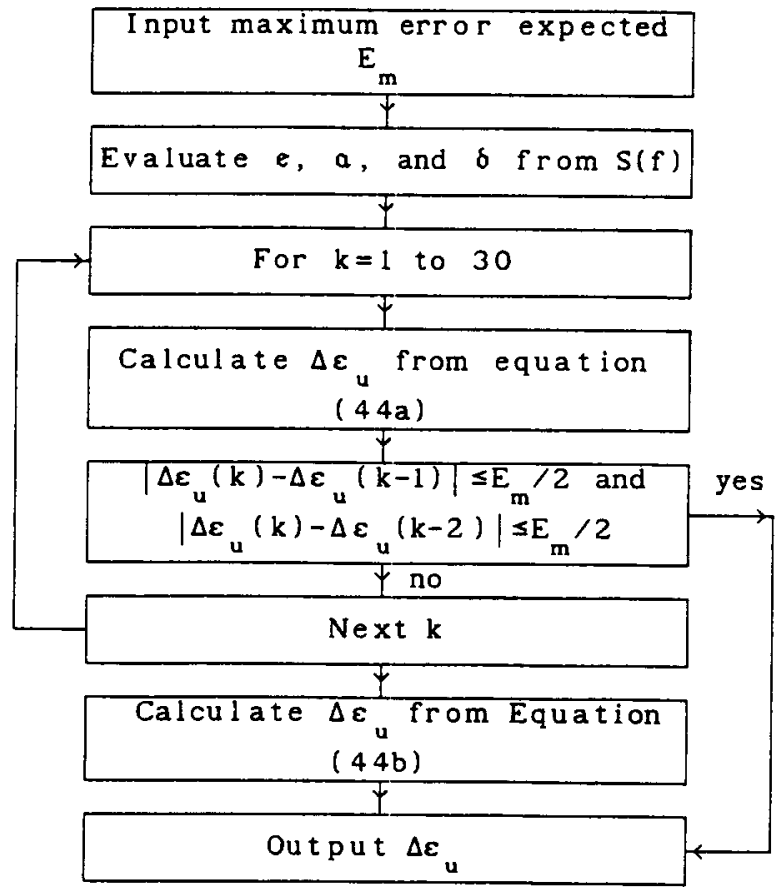

Figure 1. Flow chart for calculating the duty strain range. 


\section{MODELING FOR WAVE HISTORY DEPENDENT DAMAGE}

For a complex engineering system, a comprehensive model incorporating all history-dependent response is almost impossible. The proposed model is limited to a general stochastic system applicable to long-term sea wave statistics only. This means that the short-term sea wave statistics are treated as a stationary random process characterized by a sea wave spectrum and the damage response in one duty cycle corresponds to a process of non-ageing effect. It should be noted that if the history dependence for damage is directly introduced into the general stochastic system, then the damage response in relation with long-term sea wave process will also be a history-dependent process. In this case, a new stochastic system needs to be established since it is contradictory to the long-term sea wave process characterized by a stationary Markov process. This will lead to a very complex mathematical structure and may lose its practical signification. To retain the stochastic system characterized by a finite, stationary, and regular Markov process, a simplified model for the present investigation is proposed below.

Without the historical effect on damage from the practical stochastic system, the general stochastic system is thus a history-independent system such that the stochastic damage process can characterize the long-term sea wave process in which Markov model is valid. The history-dependent damage is considered through the modification of the damage evolution law in conjunction with the duty cycle. If we denote the modifying function as $L(H)$ where $H$ is a history dependent variable, the damage evolution law can be written as

$$
d D=L(H) \cdot f\left(\Delta \epsilon_{u}, D\right)
$$

where $f\left(\Delta \epsilon_{u}, D\right)$ is a general expression of damage evolution law which is a history independent function. It should be noted that $f\left(\Delta \epsilon_{u}, D\right)$ used here denotes a stochastic function since $\Delta \epsilon_{u}$ and $D$ are determined by a stochastic process; it is a simplified expression in which the deterministic quantities, such as mean stress, etc., do not appear.

Generally, the form of $L(H)$ is dependent on the physical and macromechanics processes under consideration. Some simplifications are necessary from the practical standpoint. A simple form proposed here considers only the effect of overloading, and postulates that there exists a restoring period induced by the overloading effect. It will further assume that damage within the restoring period is kept as constant corresponding to the beginning state of the restoring period. This implies that $L(F)$ is a step function in relation to 0 and 1 only, in which $H$ has been replaced by 4 and termed as the restoring function.

Now we define a critical sea wave state denoted by the sea state number, $N_{\mathrm{cr}}$, such that the significant effect of overloading is considered only if the sea state number beyond $N_{\mathrm{cr}}$. Consequently, $L(\xi)$ assigned to unity needs to satisfy two conditions; the current sea state number, $N_{c}$, is smaller than $N_{\mathrm{cr}}$ and the restoring period has passed.

To formulate the restoring period, we further assume that the restoring period is directly proportional to the maximum sea state number, $N_{m}$, from the start of 
the process or from the state in which the last restoring period has passed. The current restoring pe:iod in effect needs to satisfy two conditions; $N_{m} \geq N_{\mathrm{cr}}$ and the current sea state number is smaller than $N_{m}$. Furthermore, we assume that the passing rate of the restoring period is directly proportional to the current sea state number. Consequently, the restoring function li: can be expressed as

$$
\|= \begin{cases}0, & \text { for } N_{m} \leq N_{\mathrm{cr}} \text { and } N_{c}=N_{m} \\ b_{1} N_{m}-b_{2} N_{c} & \text { for others }\end{cases}
$$

where $N_{\mathrm{c}}$ is the current sea state number, and $b_{1}$ and $b_{2}$ are two constants. It should be noted that i is also a stochastic function since $N_{m}$ and $N_{c}$ are determined by a stochastic process with respect to the long-term sea wave statistics.

Using this restoring function, the step function $L\left({ }^{\prime}\right)$ can be expressed as

$$
L\left(\|^{\prime}\right)= \begin{cases}1, & \text { if } f^{\prime} \leq 0 \\ 0, & \text { if } \|^{i}>0\end{cases}
$$

This is a simple model from which the phenomena under investigation is simplified. In this model, only two constants, $b_{1}$ and $b_{2}$ need to be identified by experiment. The function l: could be modified by introducing the non-linear dependence on $N_{m}$ and $N_{c}$ if necessary.

For the sake of illustration, the applicability of this model may be described through the following example. A simulated sea state sequence of South China Sea with 10 different sea states is shown in Figure 2 as elucidated by Chow and Li [4]. If $N_{\mathrm{cr}}$ is assumed to correspond to number 8 of the sea state, then first

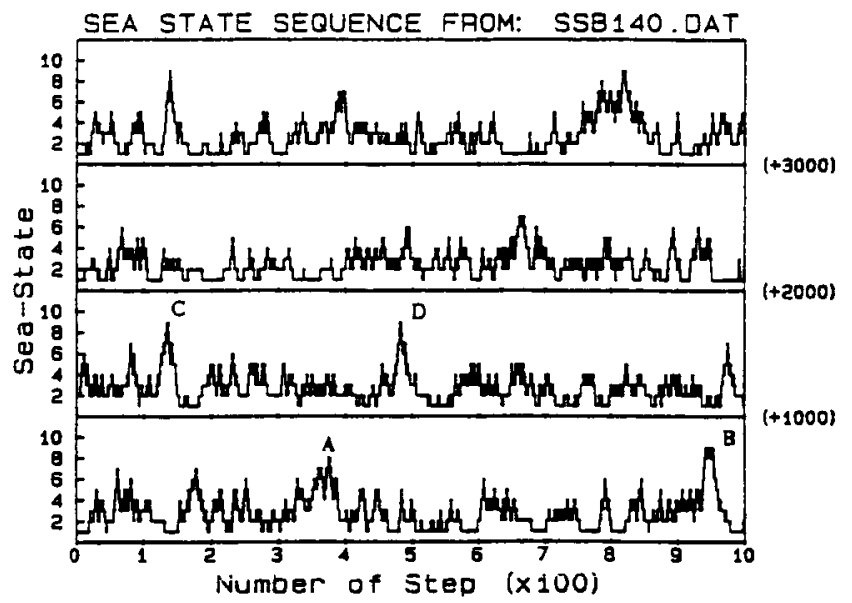

Figure 2. Simulated sea state sequence of South China Sea. 
significant overloading effect will be acting in the position $A$ of this figure. After that, the damage enters into a restoring period. Assuming $b_{1}=10$ and $b_{2}=2$, then the restoring period is $b_{1} \times N_{m}=10 \times 8=80$. In this period, the system needs to pass the states

$$
\Sigma b_{2} \cdot N_{c}=\Sigma 2 N_{c}=80
$$

without any further damage. In this sequence, the state numbers generating by Monte Carlo method are 7, 6, 5, 4, 5, 5, 6, 5*, 4, 3, 3, 2, 2, 2, 2, 3, . . . The restoring period will be over at the state number 5 with superscript $*$ since $2 \times(7+6+5+4+5+5+6+5) \geq 80$. After the sea state $5^{*}$, the damage will be accumulated continuously until the next restoring period shown in position B of Figure 2 appears. Then the overloading effect occurs in positions $\mathrm{C}, \mathrm{D}$ and so on.

\section{CONCLUSIONS}

From the practical requirements and the possibility of achieving an automated computer solution to predict the dynamic responses of offshore structures coupled with the changing/deteriorating material behaviors, the theory of damage mechanics is employed in the analysis of offshore structures. The damage coupled constitutive and evolution equations with the unified formulation for both low cycle and high cycle fatigue damage enable a consistent analysis for the structural dynamic response to be realized. The structural analysis for the whole designed period, say about 30 years, can be carried out with the aid of the proposed analytical procedure.

The proposed fatigue damage model is based on the fundamental characteristics of sea wave statistics responsible for the structural dynamic response taking into account changing or deteriorating material behaviors. An offshore structure subjected to complex ocean environment is characterized by a general stochastic system which includes a group of stochastic subsystems, each corresponding to a duty cycle. An effective analytical method has been established through the concept of duty strain range with a clear mathematical definition to achieve an analytical solution which covers all possible spectral parameters. The historydependent damage is also modeled so that the overload effects can be analyzed.

It is worth noting that the proposed damage model with its numerical procedure could be readily extended by integrating a more complex formulation of $D M$ and introducing the history-dependent damage with the restoring function $F$ described by the nonlinear one on $N_{m}$ and $N_{c}$. Moreover, the whole procedure could be fully computerized such that the practical or engineering significances deduced from the computed results can be identified for any design analysis.

\section{REFERENCES}

1. Kachanov, L. M. 1958. "Time of the Rupture Process under Creep Conditions," Ivz AN SSSR, Otd. Tech. Nauk., (8):26-31.

2. Rabotnov, Yu. N. 1963. "On the Equations of State for Creep," Progress in Appl. Mech., Prager Anniversary Volume, New York: MacMillan Corp., pp. 307-315. 
3. Hogben, N., N. M. C. Dacunda and G. F. Olliver. 1985. "Global Wave Statistics," compiled and edited by British Maritime Technology Limited.

4. Chow, C. L. and D. L. Li. 1991. "A Unified Procedure and Methodology for Generating Sea State Sequences from Globe-Wave Statistics," Int. J. of Fatigue, 13(2):17-24.

5. Pierson, W. J. and P. Holmes. 1965. "Irregular Wave Forces on a Pile," Journal of the Waterways and Harbors Division, ASCE Proc., Vol. 91, No. WW4, paper 4528, pp. 1-10.

6. Kinsman, B. 1965. Wind Waves. Englewood Cliffs, NJ: Prentice-Hall, Inc.

7. Borgman, L. E. 1967. "Spectral Analysis of Ocean Wave Force on Piling," Journal of the Waterways and Harbors Division, ASCE Proc., Vol. 93, No. WW2, paper 5247, pp. 129-156.

8. Wirsching, P. H. and P. H. Prasthofer. 1976. "Preliminary Dynamic Assessment of Deep Water Platforms," J. Structural Division, ASCE, 102 (ST7):1447-1462.

9. Irvine, N. M. 1981. "Review of Stress Analysis Techniques Used in UKOSRP," Fatigue in Offshore Structural Steels, Proc. of Conf., paper 6, London, 2.

10. Dharmavasan, S. and W. D. Dover. 1985. "Stress Distribution Formulas and Comparison of Three Stress Analysis Techniques for Tubular Joints," Trans. of ASME, 107:3.

11. Kestin, J. and J. R. Rice. 1970. "Paradoxes in Application of Thermodynamic of Strained Solids," in A Critical Review of Thermodynamics, E. B. Stuart et al., eds., Baltimore, MD: Mono Book Corp.

12. Kestin, J. and J. Bataille. 1977. "Irreversible Thermodynamics of Continua and Internal Variables," in Continuum Models of Discrete Systems," J. W. Provan, ed., Univ. of Waterloo Press.

13. Germain, P., Q. S. Nguyen and P. Suquet. 1983. "Continuum Thermodynamics," ASME $J$. of Applied Mechanics, 50:1010-1020.

14. Lemaitre, J. 1987. "Formulation and Identification of Damage Kinetic Constitutive Equations," in Continuum Damage Mechanics Theory and Applications, D. Krajcinovic and J. Lemaitre, eds., Wien, NY: Springer, pp. 37-90.

15. Chaboche, J. L. 1988. "Continuum Damage Mechanics: Part 1-General Concepts," Journal of Applied Mechanics, 55:59-64.

16. Chaboche, J. L. 1988. "Continuum Damage Mechanics: Part 2-Damage Growth, Crack Initiation, and Crack Growth," Journal of Applied Mechanics, 55:65-72.

17. Simo, J. C. and J. W. Ju. 1987. "Stress and Strain Based Continuum Damage Models-I. Formulations," Int. J. Solids Structures, 23:821-840.

18. Simo, J. C. and J. W. Ju. 1987. "Stress and Strain Based Continuum Damage Models-II. Computational Aspects," Int. J. Solids Structures, 23:841-869.

19. Chow, C. L. and J. Wang. 1987. "An Anisotropic Theory of Elasticity for Continuum Damage Mechanics," Int. J. Fract. 33:3.

20. Chow, C. L. and J. Wang. 1987. "An Anisotropic Theory of Continuum Damage Mechanics for Ductile Fracture," Engng Fract. Mech., 27:547.

21. Lu, T. J. and C. L. Chow. 1990. "On Constitutive Equations of Inelastic Solids with Anisotropic Damage," Theor. Appl. Fract. Mech. 14:187.

22. Murakami, S. 1988. "Mechanical Modeling of Material Damage," ASME J. of Applied Mechanics, 55:280-286.

23. Chow, C. L. and J. Wang. 1988. "A Finite Element Analysis of Continuum Damage Mechanics for Ductile Fracture," Int. J. of Fracture, 38:83-102.

24. Woo, C. W. and D. L. Li. 1992. "A Universal Physically Consistent Definition for Material Damage," Submitted to Int. J. of Solids Structures.

25. Woo, C. W. and D. L. Li. 1992. "Fundamental Formulation of Thermal Activation Damage Kinetics," submitted to Int. J. of Solids Structures.

26. Woo, C. W. and D. L. Li. 1992. "On Damage Kinetic Model with Atomic Activation over a System of Energy Barriers," AEPA 92, Dec. 14-17, 1992, Hong Kong.

27. Li, D. L. and C. W. Woo. 1992. "Thermodynamic Formulation for Material with Changing In- 
ternal Structures: Part I: Physical and Phenomenological Representation of Damage," Submitted to Int. J. of Solids Structures.

28. Li, D. L. and C. W. Woo. 1992. "Thermodynamic Formulation for Material with Changing Internal Structures: Part II: Deterministic Local Thermodynamic Formulation," submitted to Int. J. of Solids Structures.

29. Li, D. L. and C. W. Woo. 1992. "Thermodynamic Formulation for Material with Changing Internal Structures: Part III: Stochastic Local Thermodynamic Formulation," submitted to Int. $J$. of Solids Structures.

30. Woo, C. W. and D. L. Li. 1992. "A General Stochastic Dynamic Model of Continuum Damage Mechanics," Int. J. of Solids Structures, 29(23):2921-2932.

31. Woo, C. W. and D. L. Li. 1992. "Statistic Analysis of Material Damage with Changing Internal Structure," submited to J. of Engng. Fract. Mech.

32. Lin, Y. K. 1967. Probabilistic Theory of Structural Dynamics. New York: McGraw Hill.

33. Huston, W. B. and T. H. Skopinski. 1956. "Probability and Frequency Characteristics of Some Flight Buffet Loads," NACA TN 3733.

34. Kam, J. C. P. and W. D. Dover. 1988. "Fast Fatigue Assessment Procedure for Offshore Structures under Random Stress History," Proc. Instn. Civ. Engrs., Part 2, 85:689-700.

35. Chow, C. L. and D. L. Li. 1991. "An Analytical Solution for Fast Fatigue Assessment under Wide-Band Random Loading," Int. J. of Fatigue, 13(5):395-404. 